\title{
92 Sleep and health
}

The brain needs a sufficient amount of sleep. In this apparent resting phase, it is actually very active and regulates important body functions such as heart rate, breathing, metabolism and the immune system. Sleep is also of great importance for learning. The different sleep phases have various functions. The long-term memory necessary for specific kinds of learning, rote memorization of names, dates or learning facts, is consolidated in the deep sleep phase. By contrast, dream sleep is responsible for the procedural memory that mainly functions automatically without thinking. This covers motor processes like walking, running, swimming, jumping or riding a bicycle. Declarative and procedural capabilities are stored in different parts of the brain.

The 4 stages of sleep recorded by the electroencephalogram are the initial (falling asleep), light sleep, deep sleep (during the first half of sleep) and REM (rapid eye movement) sleep (dreaming stage). The hormone that helps regulates sleep is melatonin. This hormone is synthesized by the pineal gland which is located in the brain stem. The average weight of this pea-sized gland is only $0.1 \mathrm{~g}$. Melatonin levels are higher in younger than in older persons. The need for sleep varies among individuals. According to data gathered by the Robert Koch Institute, the average German gets between 6 and 8 hours of sleep a night.

Chronic sleep deficits lead to markedly reduced performance during the day (Lund et al. 2010, Gildner et al. 2014).
Those affected by this disorder have a five times higher risk of becoming ill than those who get enough sleep (Cappuccio et al. 2010, 2011, Hsu et al. 2012, Laugsand et al. 2014). People suffering from chronic sleep deficit will often also suffer from high blood pressure because chronic sleep deficit adversely affects endothelial function in the large blood vessels ( $\downarrow$ Chapter 51) (Gangwisch et al. 2013). One particular type of sleep disorder is snoring, a condition that other fellow human beings tend to be the ones to suffer under. Nevertheless, it can also be dangerous for the snorer themselves (2\% women, $4 \%$ men) when associated with temporary cessation of breathing (apnea). Common consequences of sleep apnea syndrome are high blood pressure, atrial fibrillation, myocardial infarction and stroke.

There should be a gap of two hours between the end of mental activity and going to bed. During this time, large meals should be avoided. Black and green tea, coffee, cola drinks and some painkillers contain sleep-impairing caffeine. Nicotine also has a stimulating effect and alcohol causes sleep-maintenance disorders. Physical

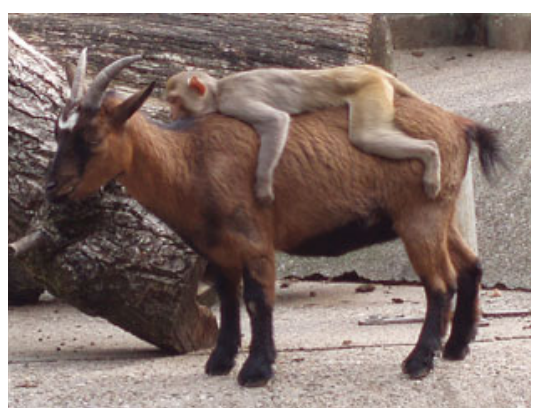

Fig. 92.1 Sleep consolidates memory activity is very helpful in promoting restful sleep (Brand et al. 2010). It causes tiredness and helps with falling asleep, although not immediately. Depending on the intensity level of the exercise, the body needs 2-4 hours to achieve full relaxation. Therefore, exercise should be avoided shortly before going to bed. 